\title{
Estabilidad del tratamiento ortodóncico-quirúrgico de avance mandibular mediante osteotomía sagital bilateral de rama mandibular en pacientes clase II. Revisión narrativa.
}

\section{Stability of the orthodontic-surgical treatment of mandibular advancement through bilateral sagittal split osteotomy in class II patients. Narrative review.}

\author{
Pablo Bórquez ${ }^{1}$, Vicente Traub ${ }^{1 *}$, Constanza Carmash ${ }^{1}$, María Ignacia Zursiedel ${ }^{1}$, \\ Juan Alberto O’Ryan ${ }^{1}$, Pedro Solé ${ }^{2}$, Carlos Vega ${ }^{3}$.
}

\author{
1. Práctica Privada, Santiago, Chile. \\ 2. Departamento de Cirugía Bucal y Maxilofacial, \\ Facultad de Odontología, Universidad de los \\ Andes, Santiago, Chile. \\ 3. Departamento de Ortodoncia, Facultad de \\ Odontología, Universidad de los Andes, Santiago, \\ Chile. \\ * Correspondencia Autor: Vicente Traub Valdés \\ | Dirección: Charles Hamilton 11, Las Condes, \\ Santiago 7550000 | Teléfono +56975475023 | \\ E-mail: vatraub@miuandes.cl \\ Trabajo recibido el 21/04/2020. \\ Trabajo revisado 24/06/2020 \\ Aprobado para su publicación el 08/07/2020
}

\begin{abstract}
RESUMEN
Introducción: La anomalía esqueletal clase II posee un 16 a 22,5\% de prevalencia mundial. Cuando estos pacientes se someten al avance mandibular mediante osteotomía sagital de rama mandibular pueden presentar un grado de inestabilidad postoperatoria evidenciándose como recidiva de éste. Objetivo: Describir la estabilidad del avance mandibular mediante osteotomía sagital bilateral de rama mandibular en pacientes clase II esqueletal. Material y método: Se realizó una búsqueda electrónica en las bases de datos PubMed, EBSCO, The Cochrane Library, Tripdatabase y Scopus mediante las palabras clave "mandibular stability", "skeletal stability", "mandibular advancement", "sagittal split osteotomy", "sagittal split ramus osteotomy", "class II", "class III" y "distraction osteogenesis", relacionadas entre sí con los términos booleanos AND, OR y NOT. También se incluyeron los términos MeSH "mandibular advancement" y "Sagittal Split Ramus Osteotomy". Paralelamente se realizó una búsqueda manual en las revistas AJODO, BJOMS, JOMS y EJO. Resultados y discusión: Se seleccionaron 29 artículos: 24 estudios observacionales analíticos, 2 revisiones sistemáticas y 3 ensayos clínicos aleatorizados. El avance mandibular mediante osteotomía sagital de rama mandibular es estable. No obstante, se debe tener en cuenta la existencia de múltiples factores pre e intraquirúrgicos que podrían generar recidiva del tratamiento.

PALABRAS CLAVE:
\end{abstract}

Avance mandibular; Osteotomía sagital de rama; Cirugía ortognática; Clase II; Estabilidad esqueletal; Estabilidad mandibular.

Int. J. Inter. Dent Vol. 14(2); 173-176, 2021.

ABSTRACT

Introduction: The class II skeletal anomaly has a $16-22,5 \%$ prevalence worldwide. When class II patients undergo mandibular advancement through Bilateral Sagittal Split Osteotomy (BSSO), they can present a postoperative instability, evidenced as a relapse. Objective: To describe the stability of mandibular advancement through BSSO in skeletal class II patients. Materials and method: An electronic search was performed in the databases PubMed, EBSCO, The Cochrane Library, Tripdatabase and Scopus using the keywords "mandibular stability", "skeletal stability", "mandibular advancement", "sagittal split osteotomy", "sagittal split ramus osteotomy", "class II", "class III" and "distraction osteogenesis", related to each other with the Boolean terms AND, OR and NOT. Also "mandibular advancement" and "Sagittal Split Ramus Osteotomy" MeSH terms were included. In parallel, a manual search in the journals AJODO, BJOMS, JOMS and EJO was performed. Results and discussion: 29 articles were selected: 24 analytic observational studies, 2 systematic reviews and 3 randomized clinical trials. Mandibular advancement through BSSO is stable. However, multiple pre and intraoperative factors that could cause a treatment relapse must be taken into account.

KEY WORDS:

Mandibular advancement; Sagittal split ramus osteotomy; Orthognathic Surgery; class II; Skeletal stability; Mandibular stability.

Int. J. Inter. Dent Vol. 14(2); 173-176, 2021. 


\section{INTRODUCCIÓN}

La anomalía dentomaxilar clase II es definida como una posición distal, esqueletal y/o dentaria, de la mandíbula con respecto al maxilar, de etiología multifactorial(1). Esto produce una serie de problemas estructurales, funcionales y/o estéticos a lo largo de la vida del paciente ${ }^{(2)}$.

Algunos pacientes clase II se pueden corregir precozmente con aparatos ortopédicos y luego con movimientos dentarios ortodóncicos, pero casos más severos, se pueden beneficiar al realizar un cambio en las relaciones esqueletales(3). Por eso, es necesario combinar e tratamiento ortodóncico y quirúrgico para lograr un resultado estable y una mejor estética(4).

Existe una serie de tratamientos ortodóncico-quirúrgicos para el tratamiento de estos casos, con distintos protocolos y técnicas, los cuales poseen múltiples factores pre, intra y postoperatorios, que afectan el pronóstico del paciente, específicamente asociado a la estabilidad esqueletal a corto y largo plazo(3).

El objetivo de esta revisión es describir la estabilidad del tratamiento ortodóncico-quirúrgico de avance mandibular mediante osteotomía sagital bilateral de rama mandibular (OSBRM) en pacientes clase II esqueletal, identificando además, los posibles factores influyentes, según la evidencia científica existente.

\section{MATERIAL Y MÉTODO}

Se realizó una búsqueda electrónica en las bases de datos PubMed, EBSCO, The Cochrane Library, Tripdatabase y Scopus mediante las palabras clave "mandibular stability", "skeletal stability", "mandibular advancement", "sagittal split osteotomy", "sagittal split ramus osteotomy", "class II", "class III" y "distraction osteogenesis", relacionadas entre sí con los términos booleanos AND, OR y NOT. También se incluyeron los términos MeSH "mandibular advancement" y "Sagittal Split Ramus Osteotomy". Paralelamente se realizó una búsqueda manual en las revistas AJODO, BJOMS, JOMS y EJO.

De los artículos obtenidos, se filtró por título, resumen y lectura a texto completo. Se seleccionaron aquellos que cumplían con los siguientes criterios de inclusión: revisiones sistemáticas, ensayos clínicos y estudios observacionales, estudios en seres humanos que incluyan pacientes clase II, sin restricción de idioma ni fecha de publicación. Fueron excluídos aquellos artículos que incluían la osteotomía vertical de rama mandibular y cirugía maxilar. Se evaluó el nivel de evidencia científica y el grado de recomendación de los artículos con las pautas del Centre for EvidenceBased Medicine de la Universidad de Oxford. Para evaluar la calidad de reporte de los artículos seleccionados se utilizaron las pautas PRISMA, CONSORT y STROBE. Para evaluar el riesgo de sesgo de los ensayos clínicos seleccionados se utilizó una modificación de la pauta propuesta por The Cochrane Collaboration y se le asignó un puntaje a cada artículo. Por último, para evaluar la ética de los artículos se consideró la existencia de consentimiento informado, la aprobación de un comité de ética y la ausencia de conflictos de interés.

\section{RESULTADOS}

Luego de haber realizado la búsqueda electrónica y manual con las estrategias mencionadas anteriormente y tras aplicar los criterios de inclusión y exclusión, 29 artículos fueron seleccionados para ser incluidos en la revisión narrativa.

Estos corresponden a 2 revisiones sistemáticas, 3 ensayos clínicos y 24 estudios observacionales analíticos.

\section{DISCUSIÓN}

La recidiva del tratamiento se podría considerar multifactorial. A continuación, se discutirán los posibles factores, mostrando su influencia en la estabilidad de la cirugía de acuerdo a lo reportado en la literatura.

\section{Tipo de fijación ósea}

En los inicios de la técnica de OSBRM (1955), para fijar los segmentos óseos se utilizaban alambres (FA) junto con la fijación intermaxilar (FIM) durante un periodo de seis a ocho semanas ${ }^{(5)}$. Sin embargo, se observó que en algunos casos esta técnica no lograba una estabilidad adecuada(6).

Para intentar mejorar la estabilidad a corto y largo plazo, se ha implementado la fijación interna rígida (FIR) en lugar de alambres, la cual puede ser llevada a cabo mediante tornillos bicorticales, tornillos monocorticales con placas de titanio o una combinación de estos ${ }^{(7)}$. Numerosos estudios han comparado la recidiva entre la FA y la FIR, encontrando resultados diversos. Los estudios de Keeling, Dolce y Van Sickels concluyen que hay una menor recidiva en los pacientes tratados con FIR en comparación a los tratados con $\mathrm{FA}^{(8-10)}$.
Por otro lado, Douma et al. compararon la estabilidad a corto y largo plazo de la FIR y la FA encontrando una recidiva no estadísticamente significativa con ninguna de las dos técnicas ${ }^{(11)}$. Resultados similares fueron encontrados por Putnam et al., sin embargo, añaden que la técnica con FIR es mejor aceptada por el paciente(12).

Watzke et al. también compararon ambas técnicas de fijación concluyendo que la mandíbula en ambos grupos tuvo excelente estabilidad, siendo mayor en el grupo con FA al lograr una posición más cercana a la postoperatoria inmediata, mientras que en el grupo con FIR, se produjo un movimiento superior y anterior de mayor magnitud durante el año de seguimiento. Los movimientos producidos en el grupo con FIR, pudieron deberse a la tensión generada por los músculos masticatorios unidos al segmento proximal, provocando una tendencia a girar hacia superior y anterior, manteniendo la posición condilar, mientras que, en el grupo con FA, la flexibilidad del alambre permitió un movimiento condilar luego del posicionamiento manual intraquirúrgico, provocando un movimiento anteroinferior revelado en el punto $\mathrm{Pg}^{(13)}$.

Otros estudios llegaron a la misma conclusión, independiente del tipo de fijación utilizada, siempre se debe esperar una cierta recidiva esqueletal y dentaria ${ }^{(14,15)}$.

\section{Tipos de fijación interna rígida}

Hoffman et al. analizaron la estabilidad de la FIR mediante tornillos bicorticales, encontrando una buena estabilidad quirúrgica a corto plazo ${ }^{(16)}$. En un estudio similar, pero con fijación mediante tornillos monocorticales y mini placas, encontraron que, en un promedio de seguimiento de 11,8 meses en 20 pacientes, en un $60 \%$ de los casos no hubo recidiva estadísticamente significativa, incluso, en algunos pacientes se registró que en el postoperatorio continuó el movimiento hacia anterior ${ }^{(17)}$.

Blomqvist et al. compararon la estabilidad con tornillos bicorticales versus tornillos monocorticales y mini placas. A los seis meses postoperatorios se encontró una recidiva sagital del punto $B$ en ambos grupos, siendo estas diferencias no estadísticamente significativas ${ }^{(18)}$.

Sato et al. evaluaron 3 métodos de FIR: 4 tornillos monocorticales y mini placas, 3 tornillos bicorticales y una técnica híbrida con 4 tornillos monocorticales, mini placas y un tornillo bicortical. Al evaluar sagitalmente a los 6 meses, se evidenció una mayor estabilidad en el grupo de tornillos monocorticales y mini placas, seguido por el grupo de tornillos bicorticales, en el cual hubo una tendencia al avance mandibular, y por último en la técnica híbrida, a pesar de ser mínimos, se registraron cambios hacia posterior. Las diferencias no fueron estadísticamente significativas ${ }^{(7)}$.

Una revisión sistemática donde se comparó el uso de fijación con tornillos bicorticales y monocorticales con mini placas, se registró una mayor tasa de recidiva al utilizar tornillos bicorticales a largo plazo, pero estos resultados se podrían deber a que no se incluyeron estudios con tornillos monocorticales a largo plazo, los que podrían evidenciar el mismo grado de recidiva ${ }^{(19)}$.

\section{Magnitud del avance mandibular}

Will y West $^{(20)}$ evaluaron la recidiva en relación al grado de avance mandibular, utilizando fijación con alambre. Se obtuvieron recidivas más altas en pacientes sometidos a un avance mandibular mayor ${ }^{(6)}$. En aquellos pacientes en que se realizaron movimientos mayores, se soportan tensiones musculares paramandibulares más grandes. $Y$ al utilizar alambres, estos reciben las fuerzas en distintas direcciones, por lo que tienden a volver a su posición original.

En otros estudios donde se realizaron movimientos quirúrgicos pequeños asociados a FIR, se logró una estabilidad adecuada con leve recidiva. Esta no tuvo relación estadísticamente significativa con el grado de avance mandibular, ya que la tracción de los tejidos circundantes hacia posterior fue menor ${ }^{(21-24)}$

Otros estudios donde se utilizó FIR, llegaron a la conclusión de que la recidiva era directamente proporcional a la magnitud del avance mandibular cuando se realizaban avances de más de $8.5 \mathrm{~mm}$. Sin embargo, cuando el avance fue menor a $5 \mathrm{~mm}$, al cabo de 3 años se observaron movimientos mandibulares hacia anterior ${ }^{(7,25,26)}$.

\section{Ángulo de plano mandibular}

Una serie de estudios han evaluado cómo puede influir el ángulo de plano mandibular preoperatorio de un paciente al momento de realizar la cirugía. En algunos estudios donde se evaluó a los pacientes según el plano mandibular, no encontraron diferencias estadísticamente significativas en la recidiva horizontal y vertical de la posición mandibular post quirúrgica $(21,27,28)$.

Otro estudio comparó la estabilidad en pacientes tratados con FIR y los agruparon según el ángulo de plano mandibular. En el grupo de ángulo alto se obtuvo a los tres años una recidiva de $36 \%$ del avance quirúrgico. 
En el grupo de ángulo bajo se registró una recidiva de $27,6 \%$ del avance quirúrgico, y disminución de la altura facial anterior. En ambos grupos hubo una pequeña disminución de la altura facial posterior. Con respecto a los movimientos rotacionales postoperatorios, en el grupo de ángulo alto, se observó una rotación del segmento proximal en sentido horario siendo estadísticamente significativo con respecto al otro grupo ${ }^{(29)}$.

Blomqvist et al. registraron grandes cambios postoperatorios verticales hacia superior a nivel de $\mathrm{Pg}$ en pacientes de ángulo bajo, siendo estadísticamente significativos al compararlos con el grupo de ángulo alto(18). Esto se explica, ya que los pacientes con ángulo bajo generalmente presentan mordidas profundas que necesitan un movimiento quirúrgico marcado hacia inferior y anterior para disminuir el overbite y overjet, aumentando la estabilidad oclusal, sin embargo, en el postoperatorio este tiende a recaer a su posición inicial, debido a la tensión y orientación de la musculatura( ${ }^{(30)}$.

Elángulo de plano mandibular se ha visto relacionado con la reabsorción condilar progresiva ${ }^{(28,31,32)}$. Xi et al. registraron cóndilos de mayor volumen en pacientes de ángulo bajo. Dentro de los casos que hubo reabsorción condilar, los pacientes de ángulo bajo presentaron menor disminución de su volumen condilar luego de un año postoperatorio, evidenciando menor recidiva esquelética horizontal y vertical en comparación con los pacientes de ángulo alto con reabsorción condilar ${ }^{(31)}$. Esto indica que el ángulo de plano mandibular es un factor indirecto en este caso, ya que la recidiva es producida por la reabsorción condilar ${ }^{(33)}$.

\section{Reabsorción condilar postoperatoria (RCP)}

Varios estudios observaron una relación directa estadísticamente significativa entre la disminución de la longitud de rama mandibular posiblemente asociada a RCP y la recidiva esquelética ${ }^{(28,32)}$

$\mathrm{Xi}$ et al. concluyeron que la disminución del volumen condilar se asocia con un aumento de la recidiva esquelética en el punto $\mathrm{Pg}$, una disminución de la altura facial posterior y un aumento del ángulo de plano mandibular ${ }^{(31)}$. Sin embargo otros estudios encontraron que no existe relación entre la reabsorción condilar progresiva y la recidiva esqueletal $(34,35)$.

Los resultados encontrados no llegan a un consenso sobre la influencia de la RCP en la recidiva esqueletal|(33, 35-38).

\section{Edad}

Un estudio que evaluó la estabilidad de pacientes entre 17 y 30 años, no encontró una relación entre la recidiva post operatoria y la edad de los pacientes al momento de la cirugía ${ }^{(39)}$. Resultados similares se encontraron en otro estudio que evaluó pacientes entre 18 y 28 años ${ }^{(25)}$. Otros estudios que evaluaron pacientes entre 14 y 17 años, tampoco encontraron diferencias estadísticamente significativas entre la edad y la recidiva ${ }^{(22,26)}$.
Por otro lado, un estudio realizado por den Besten et al. evaluó la estabilidad en pacientes menores de 18 años, donde algunos aún no había terminado el crecimiento, comparado con un grupo control de pacientes de 20 a 24 años, que ya habían terminado su crecimiento craneofacial. Según el último registro cefalométrico, la recidiva horizontal y vertical fue similar en ambos grupos, sin encontrar una relación estadísticamente significativa entre las recidivas registradas y las edades de los pacientes, incluso ocurrió más recidiva en el grupo control $(16,4 \%)$ que en el grupo adolescente $(10,9 \%)$. Existe una posible explicación a esta diferencia, la cual podría ser por el crecimiento postoperatorio que ocurre luego de la cirugía, ya que a pesar de que ambos grupos manifestaron recidiva, en el grupo adolescente pudo haberse compensado por el crecimiento mandibular en la misma dirección que el movimiento quirúrgico ${ }^{(40)}$

Por el contrario, Blomqvist et al. encontraron resultados discordantes. Los pacientes se agruparon en tres grupos: menores de 20 años, entre 20 y 40 años y mayores de 40 años, encontrando una mayor estabilidad en los pacientes del último grupo ${ }^{(18)}$.

\section{CONCLUSIONES}

Existen múltiples factores a considerar al momento de evaluar la estabilidad post operatoria de la osteotomía sagital de rama para avance mandibular.

La fijación interna rígida es capaz de evitar grandes cambios postoperatorios y otorga mayor comodidad al paciente. Sin embargo, no hay consenso en la evidencia disponible, sobre la existencia de diferencias estadísticamente significativas con la fijación con alambres.

Cuando es necesario un avance mandibular de mayor magnitud en pacientes con ángulo de plano mandibular divergente, se debe esperar una mayor recidiva. Esto debido a que se requiere un mayor movimiento antihorario, generando grandes tensiones musculares en sentido contrario al avance.

No se encontró relación entre la reabsorción condilar postoperatoria y la estabilidad del avance mandibular.

En pacientes previo al peak puberal se ha observado un mejor resultado post operatorio debido al crecimiento residual que ocurriría en la dirección del avance. Sin embargo, se necesitan estudios que evalúen este factor a largo plazo para evidenciar posibles recidivas luego de finalizar el crecimiento craneofacial.

Faltan ensayos clínicos aleatorizados con mayor tamaño muestral para sacar conclusiones extrapolables de todos estos factores.

\section{CONFLICTO DE INTERESES}

Los autores declaran no presentar conflictos de interés.

Este trabajo no cuenta con financiamiento alguno.

\section{Bibliografía}

1. Bishara SE. Class II Malocclusions: diagnostic and clinical considerations with and without treatment. Semin Orthod. 2006 Mar;12(1):11-24.

2. Escriván De Saturno LD, Torres C M. Ortodoncia en dentición mixta, Amolca 2007; p.562

3. Quevedo Rojas LA. Osteotomía sagital de rama mandibular en cirugía ortognática. Rev Esp Cir Oral Maxilofac. 2004 Feb;26(1):14-21

4. Donjuán Villanueva JJ, Vásquez Estrada HA, Hernández Carvallo JR, Nachón García MG. Tratamiento ortodóncico-quirúrgico en paciente con maloclusión clase II. Reporte de caso. Rev Mex Ortod. 2016 Abr;4(2):88-95.

5. Reitzik M. Cortex-to-cortex healing after mandibular osteotomy. J Oral Maxillofac Surg. 1983 Oct:41(10):658-63.

6. Will LA, West RA. Factors influencing the stability of the sagittal split osteotomy for mandibular advancement. J Oral Maxillofac Surg. 1989 Aug;47(8):813-8.

7. Sato FRL, Asprino L, Fernandes Moreira RW, de Moraes M. Comparison of postoperative stability of three rigid internal fixation techniques after sagittal split ramus osteotomy for mandibular advancement. J Cranio-Maxillofac Surg. Jul 2014;42(5):e224-9

8. Keeling SD, Dolce C, Van Sickels JE, Bays RA, Clark GM, Rugh JD. A comparative study of skeletal and dental stability between rigid and wire fixation for mandibular advancement. Am J Orthod Dentofacial Orthop. 2000 Jun;117(6):638-49.

9. Van Sickels JE, Flanary CM. Stability associated with mandibular advancement treated by rigid osseous fixation. J Oral Maxillofac Surg. 1985 May;43(5):338-41.

10. Dolce C, Van Sickels JE, Bays RA, Rugh JD. Skeletal stability after mandibular advancement with rigid versus wire fixation. J Oral Maxillofac Surg. 2000 Nov;58(11):1219-27.

11. Douma E, Kuftinec MM, Moshiri F. A comparative study of stability after mandibular advancement surgery. Am J Orthod Dentofacial Orthop. 1991 Aug;100(2):141-55.

12. Putnam GD, Bouwman JPB, Tuinzing DB. Stability of the osteotomy site following bilateral sagittal split osteotomy: screw fixation $v$ IMF. $\mathrm{Br} \mathrm{J}$ Oral Maxillofac Surg. 1993 Aug;31(4):213-6.
13. Watzke IM, Turvey TA, Phillips C, Proffit WR. Stability of mandibular advancement after sagittal osteotomy with screw or wire fixation: A comparative study. J Oral Maxillofac Surg. 1990 Feb;48(2):108-21.

14. Moen K, Wisth PJ, Skaale S, Bøe OE, Tornes K. Dental or skeletal relapse after sagittal split osteotomy advancement surgery? long-term follow-up. J Oral Maxillofac Surg. 2011 Nov;69(11):e461-8.

15. Berger JL, Pangrazio-Kulbersh V, Bacchus SN, Kaczynski R. Stability of bilateral sagittal split ramus osteotomy: Rigid fixation versus transosseous wiring. Am J Orthod Dentofacial Orthop. 2000 Oct;118(4):397-403.

16. Hoffman GR, Moloney FB. The stability of facial osteotomies. 2. Mandibular advancement with bicortical screw fixation. Aust Dent J. Aug 1995;40(4):213-9.

17. Abeloos J, De Clercq C, Neyt L. Skeletal stability following miniplate fixation after bilateral sagittal split osteotomy for mandibular advancement. J Oral Maxillofac Surg. 1993 Apr;51(4):366-9.

18. Blomqvist JE, Ahlborg G, Isaksson S, Svartz K. A comparison of skeletal stability after mandibular advancement and use of two rigid internal fixation techniques. J Oral Maxillofac Surg. 1997 Jun;55(6):568-74.

19. Joss CU, Vassalli IM. Stability after bilateral sagittal split osteotomy advancement surgery with rigid internal fixation: a systematic review. J Oral Maxillofac Surg. Feb 2009;67(2):301-13

20. Fish LC, Epker BN. Prevention of relapse in surgical-orthodontic treatment. Part 1. Mandibular procedures. J Clin Orthod. 2986 Dec;20(12):826-41.

21. Hartlev J, Godtfredsen E, Andersen NT, Jensen T. Comparative study of skeletal stability between postoperative skeletal intermaxillary fixation and no skeletal fixation after bilateral sagittal split ramus osteotomy: an 18 months retrospective study. J Oral Maxillofac Res. 2014 Apr;5(1);e2

22. Kirkpatrick TB, Woods MG, Swift JQ, Markowitz NR. Skeletal stability following mandibular advancement and rigid fixation. J Oral Maxillofac Surg. 1987 Jul;45(7):572-6. 23. Van Sickels JE, Larsen AJ, Thrash WJ. A retrospective study of relapse in rigidly fixated sagittal split osteotomies: Contributing factors. Am J Orthod Dentofacial Orthop. 1988 May;93(5):413-8. 
24. McDonald WR. Stability of mandibular lengthening: a comparison of moderate and large advancements. Oral Maxillofac Surg Clin North Am. 1990 Jan;2(1):729-35. 25. Tabrizi R, Nili M, Aliabadi E, Pourdanesh F. Skeletal stability following mandibular advancement: is it influenced by the magnitude of advancement or changes of the mandibular plane angle? J Korean Assoc Oral Maxillofac Surg. 2017 Jun;43(3):152. 26. Kierl MJ, Nanda RS, Fräns Currier G. A 3-year evaluation of skeletal stability of mandibular advancement with rigid fixation. J Oral Maxillofac Surg. 1990 Jun;48(6):587-92.

27. Storms AS, Miclotte A, Grosjean L, Cadenas de Llano-Pérula M, Alqerban A, Fieuws S, et al. Short-term hard and soft tissue changes after mandibular advancement surgery in Class II patients: a retrospective cephalometric study. Eur J Orthod. 2017 Oct;39(5):567-76.

28. Eggensperger N, Smolka K, Luder J, lizuka T. Short- and long-term skeletal relapse after mandibular advancement surgery. Int J Oral Maxillofac Surg. 2006 Jan;35(1):36-42.

29. Mobarak KA, Espeland L, Krogstad O, Lyberg T. Mandibular advancement surgery in high-angle and low-angle class II patients: Different long-term skeletal responses. Am J Orthod Dentofacial Orthop. 2001 Apr;119(4):368-81.

30. Proffit WR, Turvey TA, Phillips C. Orthognathic surgery: a hierarchy of stability. Int J Adult Orthodon Orthognath Surg. 1996 Jan;11(3):191-204

31. Xi T, Schreurs R, van Loon B, de Koning M, Bergé S, Hoppenreijs T, et al. 3D analysis of condylar remodelling and skeletal relapse following bilateral sagittal split advancement osteotomies. J Cranio-Maxillofac Surg. 2015 May;43(4):462-8.

32. Motta AT, Cevidanes LHS, Carvalho FAR, Almeida MAO, Phillips C. Threedimensional regional displacements after mandibular advancement surgery: one year of follow-up. J Oral Maxillofac Surg. 2011 May;69(5):1447-57.
33. Arnett GW, Milam SB, Gottesman L. Progressive mandibular retrusionidiopathic condylar resorption. Part I. Am J Orthod Dentofacial Orthop. 1996 Jul;110(1):8-15.

34. Franco AA, Cevidanes LHS, Phillips C, Rossouw PE, Turvey TA, Carvalho F de $A R$, et al. Long-term 3-dimensional stability of mandibular advancement surgery. $J$ Oral Maxillofac Surg. 2013 Sep;71(9):1588-97.

35. Kobayashi T, Izumi N, Kojima T, Sakagami N, Saito I, Saito C. Progressive condylar resorption after mandibular advancement. Br J Oral Maxillofac Surg. 2012 Mar:50(2):176-80.

36. Cutbirth M, Van Sickels JE, Thrash WJ. Condylar resorption after bicortical screw fixation of mandibular advancement. J Oral Maxillofac Surg. Feb 1998;56(2):178-82. 37. Hwang S-J, Haers PE, Zimmermann A, Oechslin C, Seifert B, Sailer HF. Surgica risk factors for condylar resorption after orthognathic surgery. Oral Surg Oral Med Oral Pathol Oral Radiol Endod. May 2000;89(5):542-52.

38. Hoppenreijs TJM, Freihofer HPM, Stoelinga PJW, Tuinzing DB, van't Hof MA. Condylar remodelling and resorption after Le Fort I and bimaxillary osteotomies in patients with anterior open bite. Int J Oral Maxillofac Surg. Apr 1998;27(2):81-91. 39. Joss CU, Thuer UW. Stability of the hard and soft tissue profile after mandibular advancement in sagittal split osteotomies: a longitudinal and long-term follow-up study. Eur J Orthod. Oct 2008;30(1):16-23.

40. den Besten CA, Mensink G, van Merkesteyn JPR. Skeletal stability after mandibular advancement in bilateral sagittal split osteotomies during adolescence. J Cranio-Maxillofac Surg. Jul 2013;41(5):e78-82. 